\title{
Genre and Online Games
}

\section{Comment 2: Romance, Dancing games are not online.}

\section{We've tried to keep our entry focused on online games rather than genre and games more generally. The asian influence on online games does not create nearly so much novelty as their impact on other genres.}

Tanya Krzywinska and Douglas Brown

\begin{abstract}
Genre is used by game developers as a means of targeting certain markets providing easily-identified product differentiation. This entry provides an overview of the development and diversity of genre in online games and maps out the major factors that drive genre formations. In addition, academic approaches to the study of genre are addressed in conjunction with the commercial and cultural contexts that shape online game genres.
\end{abstract}

\section{Keywords}

Genre; Gameplay; Hybridity; Players; Casual; Massively Multiplayer Online Games; RPG; Text.

Having found their feet on the margins of the game industry, games played online now occupy prime territory. They are no longer simply the preserve of the humble text parser interface, as they were at the birth of the internet when Multi-User-Dungeons (MUDs) proved the concept of playing games online with others. Nor are they confined mainly to the Massively Multiplayer Role-Playing Game (MMORPG), as was the case in the early 2000s. The online game now comes in many different generic guises, from large sprawling world-based games to those of much smaller scale. Some are designed to draw in players from a range of non-traditional markets. Some demand considerable time investments from players, others less so. Many are subscription-based, while others are free-to-play offering in game items that can be bought using a credit card.=

There are patterns and trends in genre preference; age, gender as well the influence of broader trends for example Peter Jackson's Lord of the Rings films contributed to whetting an appetite for fantasy-based RPG games; Zagal and Tomuro (2013) note the influence of culture on game genre preferences, arguing that Japanese games are accepted by Western audiences: 'The global appeal that many Japanese videogames enjoy may be due to the fact that they've been carefully designed and developed to be culturally neutral, rather than particularly Japanese"While also considering whyJapanese players tend to reject western designed games : "Japanese game developer Atsuhi Inaba notes how [western games] "quickly came to equal shooting games" and that "to be honest, very few people know about Gears Of War or Mass Effect or even Call Of Duty in Japan"'.

As a result of commercial success and their widely accessible presence in social networks, the large-scale move towards social online games has necessitated some significant changes to the types of games made and invested in. In addition to the growing number of large-scale multiplayer games, we can outmanoeuvre and outshoot teams of other players in the Call of Duty franchise, mow down zombies with a small group of friends in squad-based games such as Left 4 Dead (Valve, 2008), play board and card 
games such as Ticket to Ride (Days of Wonder, 2011) and Dominion (Goko, 2013) with our friends and family, or have our fellow facebookers help us tend our crops and animals in Farmville. Increased opportunities for online distribution alongside the games industry's considerable efforts to widen the potential markets for games has led to an explosion of diversity in the genre of online games: genre mashups such as Amateur Surgeon Hospital (Mediatonic, 2012), old genres renewed through multiplayer access such as Final Fantasy XI and XIV(Square-Enix 2002 and 2012), Orcs Must Die 2 (Robot

Entertainment, 2012) and Dungeon Defenders (Trendy Entertainment, 2010) which revitalise tower defence gameplay, alongside games that are creating new genres such as Draw Something (Omgpop, 2012).

Revolutions in established genres also result from this vigorous diversity. Demons' Souls (From Software, 2009) brought the single-player RPG into online space, creating new a sense of dread by introducing the constant threat of invasions into your game from other players. New Super Mario Bros. $U$ (Nintendo, 2012) has a social online presence, asking players to leave reviews of levels to be displayed during loading screens for the rest of the game's community. It is a rare game now which does not include any kind of online feature, even if that extends only as far as broadcasting achievements to players' friends lists on the hosting platform. Several of the games mentioned so far do not have to be played online at all, but they are primarily designed with this in mind. Modern military shooters since Call of Duty: Modern Warfare (Infinity Ward, 2007) have had single-player campaign modes, but the reason why these games sell so well is the multiplayer experience, and the single-player element is generally seen as providing training for the 'real' online game. This angle is so entrenched in the military FPS genre that publishers insist upon an online mode being present, even when games such as Spec Ops: The Line (Yager, 2012) focus on the single-player campaign and its narrative dimension.

Online games now come in many different generic guises and are accessed using a variety of interfaces and devices. It follows therefore that while 'online' games were formerly considered a genre in their own right, just being found online is no longer enough to be a prime generic marker in marketing rhetoric. This sea change has yet to be analysed in detail by game scholars. It is how a game's type is ascertained, agreed upon or indeed disputed, possibly changed over time, and how that categorisation becomes used, rejected or replaced by players that is important when theorising the online game in terms of genre. Academic approaches to genre within the Humanities tend to gravitate around five poles: textual characteristics that a set of artefacts have in common; the changes to genre that occur historically; the commercial use of generic labelling to brand a product for an audience; the ways in which audiences use and understand genre; the persuasive, ideological intention of a given genre.

All these poles should inform our understanding of genre and online games, thereby shifting any analysis beyond the confines of a narratological understanding of game genre from which it has historically stemmed. Ric Altman (1999) argues that it is essential to consider genre as at once a set of evolving textual strategies placed in a commercial context, as well as an interpretational and ideological process, if we are to apprehend its complexity and be better able to theorise and historicise it. Altman's expansive prescription does not though take into account the particular issues arising in the case of genre and online games. Even in the most straight-forward approach to genre as textual property, genre in the sphere of games is even more complex than in other fields such as film, TV or popular literature. Genre does not refer just to theme and narrative formation. It is also used by industry, press and players alike to refer to the principal features of gameplay activity (role-play, shooter, puzzle) or the primary perspective 
used (first or third person). Tom Apperley holds that gameplay features should be focused on exclusively, calling them a 'genre of interactivity'. $(2006, \mathrm{p} .7)$

Added to gameplay and perspective are other commonly used generic terms for the size of group a game is made for (squad, massively multiplayer, multiplayer, team etc). Aki Järvinen (2002) argues that such hybridity characterises game genres as "complex sums of interaction and rule mechanisms, audiovisual styles and popular fiction genre conventions". One particular element which has to be added to this definition of game genre as both hybrid and as a means of market differentiation in the context of online games is player skill. The terms 'casual' and 'hardcore' have increasingly become important and informative genre markers, also noted by Felix Raczkowski $(2012$, p.60) in relation to shifting patterns of genre in games. This nomenclature emerged as the audiences games are developed for broadened and it expressly addresses players in terms of skill or preferred play style. 'Casual' in particular is now arguably a genre in its own right, especially in social online spaces such as Facebook and in the arena of 'freemium' internet browser-based games.

Despite the presence of all these generically resonant, game-media specific markers, there are also markers from other media in play. So while an MMORPG is regarded as a genre in its own right, a game included in this genre will also draw on the thematic qualities and narrative formations of other existing genres established outside games. World of Warcraft (Blizzard, 2004-present) and The Secret World (Funcom, 2012-present) are both known as MMORPGs, where players build characters by accumulating power by running quests, yet the former uses the vernacular of Fantasy while the latter uses that of Horror and The Weird Tale. This has lead King and Krzywinska to the claim that extra-gamic genre is best thought of as providing 'milieu' for the more procedural aspects of a game $(2002$, p. 27). Such ubiquitous generic milieu are often mobilised to render a product recognisable to potential purchasers in terms and images easily communicated in marketing material. Familiarity and the evocation of previous good playing experiences is core to the prevalence of genre as a marketing tool, as Steve Neale (2000) has argued in relation to film. All the generic over-determination of online games comes about because many games seek to dovetail particular game system types and structures together with characteristics of existing genres from other media. Endogenous and exogenous generic formations have their own distinctive agendas. The mash-up of the two is not always successful, and some genres fit together more seamlessly than others. Science Fiction, Fantasy, Mystery/Detective and Horror are core staples which suit best the ludic agendas of game systems. Not all popular and successful games use these however. One such example is Farmville (Zynga, 2009-present), currently the most widely played social-based online game. This game remixes building and quest gameplay features, allowing players to build up a farm with the help of their friends.

There are other games that do not make use of the trappings of existing genres, endogenous or exogenous. These games seek the difficult task of escaping the confines of generic regularity as is the case with Journey, which treats its online nature in a way that differs from previous games, eschewing usernames, servers and lobbies. It uses online elements to ensure that no two playthroughs are ever alike.

It is important to note that the definition of genre is itself placed under stress by the burden of all this complexity. As with other media such as film, different definitions and uses of genre serve the interests of different parties and might be said to operate as a form of rhetoric. The genre choices made by players may be regarded as performative of identity, displaying sub-cultural capital perhaps, or signifying 
'distinction' in the sense that Pierre Bourdieu (2010) has outlined at work generally in consumer culture. It is useful to cite Bourdieu here as his work places focus on the ways in which formal features come into being, rather than on these features themselves. For Bourdieu, preferences towards certain types of genres are formed socially rather than textually (see Kirkpatrick, 2011), visible in the generalised differences in game genre preference between Western and Japanese markets.

Game culture is especially sensitive to nuances of taste and preference as a means of performing identity online; this resonates with the competitive nature of many popular online games, where in some genres player-versus-player combat, and the comparing of skills that it necessitates is a staple. The genres of games played can easily become badges of honour to some players, hence the vocal antipathy of 'hardcore' gamers towards casual gaming. Through the structural force of the binary opposition, the term 'hardcore' finds its definition and strength through that which becomes deemed as 'casual'. This distinction also demonstrates a gender distinction at work in the rhetorics of game culture and genre. It is clear therefore that genre in online games is far from a 'Platonic' category (Altman, 1999). It is instead a powerful lure, dividing the market commercially and culturally and often separating players in terms of age, gender and taste. Simultaneously textual, rhetorical, social, and commercial, the meaning of genre in onlines games is very far from uncontested and clear. As such it warrants much closer attention from scholars and from researchers as a topic in its own right.

\section{Bibliography}

Ric Altman, Film/Genre. BFI, 1999.

Pierre Bourdieu, Distinction. London: Routledge, 2010.

Thomas H. Apperley., Genre and Game Studies: toward a Critical Approach to Video Game Genres Simulation and Gaming vol.37 no.1 pp.6-23, March 2006. Sage.

Aki Järvinen 'Halo and the anatomy of the FPS' Game Studies vol.2 no.1, July 2002. Sage. [Online] Available at: < http://www.gamestudies.org/0102/jarvinen/ > [Accessed April 2nd 2013]

Geoff King and Tanya Krzywinska (eds.) ScreenPlay cinema/videogames/interfaces. Wallflower Press: London/NY, 2002.

Graeme Kirkpatrick Aesthetic Theory and the Videogame Manchester University Press: Manchester, 2011 Steve Neale. Genre and Hollywood. Routledge: London/NY, 2000.

Felix Raczkowski “"And What Do You Play?": A Few Considerations Concerning a Genre Theory' in J. Fromme and A. Unger (eds.) Computer games and New Media Cultures: A Handbook of Digital Game Studies. Springer: Heidelberg/NY/London. 


\section{Gameography (in case it is needed)}

Amateur Surgeon Hospital (Mediatonic, PC, 2012)

Call of Duty: Modern Warfare (Infinity Ward, PC, 2007)

Demons' Souls (From Software, PS3, 2009)

Dominion (Goko, PC, 2013)

Draw Something (Omgpop, Iphone, 2012)

Dungeon Defenders (Trendy Entertainment, Iphone, 2010)

Farmville (Zynga, PC, 2009)

Journey (Thatgamecompany, PS3, 2012).

Left 4 Dead (Valve, PC, 2008)

New Super Mario Bros. U (Nintendo, Wii U, 2012)

Orcs Must Die 2 (Robot Entertainment, PC, 2012)

Spec Ops: The Line (Yager, PC, 2012)

The Secret World (Funcom, PC, 2012)

Ticket to Ride (Days of Wonder, Ipad, 2011)

World of Warcraft (Blizzard, PC, 2004) 